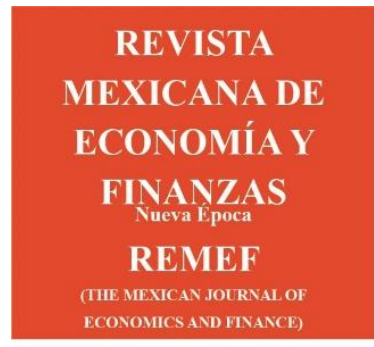

Revista Mexicana de Economía y Finanzas, Nueva Época

Volumen 17 Número 1, Enero - Marzo 2022, pp. 1-13, e708

DOI: https://doi.org/10.21919/remef.v17i1.708

(Recibido: 23/marzo/2020, aceptado: 9/septiembre/2020, publicado: 4/octubre/2021)

\title{
¿Impulsan la actividad económica en América Latina el número de sucursales bancarias y cajeros automáticos?
}

\author{
Ana Lilia Hernández Pineda - Universidad Autónoma del Estado de Hidalgo, México \\ Alí Aali-Bujari ${ }^{1}$ - Universidad Autónoma del Estado de Hidalgo, México
}

El objetivo de ésta investigación es analizar el impacto del número de sucursales bancarias por cada 100000 adultos y cajeros automáticos por cada 100000 adultos en el crecimiento económico en Argentina, Brasil, Chile, Costa Rica, Ecuador, México, Perú y Uruguay, durante 2004-2018. Metodología: se estiman modelos de datos panel: efectos fijos y efectos aleatorios. Resultados: El principal hallazgo, es que el crecimiento económico es afectado positivamente por el número de sucursales bancarias y de cajeros automáticos. Recomendaciones: Se exhorta a los tomadores de decisiones buscar los instrumentos e incentivos adecuados para aumentar el número de sucursales bancarias y cajeros por cada 100000 adultos, y así contribuir al crecimiento económico. Limitaciones: La investigación se restringe a analizar sólo el rol del número de sucursales bancarias y cajeros automáticos en el crecimiento económico. Originalidad: se concentra en siete economías de América Latina, se tiene una mayor disponibilidad de datos, se realiza análisis de datos panel, que permite utilizar mayor cantidad de países, variables y períodos. Conclusiones: El número de sucursales bancarias y cajeros automáticos impulsan la actividad económica en América Latina.

Clasificación JEL: 010, 031, 047.

Palabras clave: Crecimiento económico, desarrollo financiero, datos panel.

\section{Does the Number of Bank Branches and ATMs Boost Economic Activity in Latin America?}

This research is aimed at analyzing the impact of the number of bank branches and the number of ATMs on economic growth in Argentina, Brazil, Chile, Costa Rica, Ecuador, Mexico, Peru and Uruguay, during 2004-2018. Methodology: panel data models are estimated: fixed effects and random effects. Results: The main finding is that economic growth is positively affected by the number of bank branches and ATMs. Recommendations: Decision makers are encouraged to seek the appropriate instruments and incentives to increase the number of bank branches and ATMs per 100,000 adults, and thus contribute to economic growth. Limitations: The research is restricted to analyzing only the role of the number of bank branches and ATMs in economic growth. Originality: It is concentrated in seven Latin American economies, there is greater data availability, panel data analysis is carried out, which allows the use of more countries, variables and periods. Conclusions: The number of bank branches and ATMs drive economic activity in Latin America.

JEL Classification: 010, 031, 047.

Keywords: Economic growth, financial development, panel data.

\footnotetext{
${ }^{1}$ Autor de correspondencia. Universidad Autónoma del Estado de Hidalgo, Pachuca-Actopan Km 4.5, Campo de Tiro, 42039, Pachuca de Soto, Hidalgo, México. Teléfono: +527717172000 ext. 5801. alibujari@yahoo.es. ORCID: http://orcid.org/00000002-7392-6635

* Sin fuente de financiamiento para el desarrollo de la investigación
} 


\section{Introducción}

La relación entre el desarrollo financiero y el crecimiento económico ha sido objeto de estudio de diferentes investigaciones, tanto teóricas como empíricas, a lo largo del tiempo: Schumpeter (1954) encuentra que el sector financiero es clave para impulsar la innovación tecnológica y por ende al desarrollo económico. El desarrollo empresarial promueve el crecimiento económico y este a su vez, estimula la creación de nuevos instrumentos y mecanismos de financiación. Asimismo, Wicksell (1934) argumenta que la demanda de crédito dinamiza la economía, encuentra que el sector financiero juega un papel crucial para financiar la inversión, la innovación, el progreso tecnológico, y en este sentido contribuye al crecimiento económico. Por otro lado, Goldsmith (1969) encuentra una relación positiva entre el desarrollo financiero y el crecimiento económico, utilizando el análisis de corte trasversal, para una muestra de 35 países.

Aunado a lo anterior, el desarrollo financiero debe considerase bajo el enfoque de crear y expandir las instituciones, mercados y productos financieros que promuevan la inversión y el crecimiento. El desarrollo financiero es función de intermediación que llevan a cabo los mercados financieros entre los ahorradores e inversionistas en el proceso para impulsar el desarrollo económico. Existe una gran cantidad de estudios realizados en muchos países respecto a la relevancia del sector financiero para el crecimiento económico: Mckinon (1973), King y Levine (1993), Bencivenga (1996), Levine y Zervos (1998), Rajan y Zingales (1998), Venegas-Martínez (1999), Beck y Levine (2002), Nazmi (2005), Guidotti (2007), Brianto (2010), López y Rodríguez (2010), Bittecourt (2012), Tabak et al. (2013), Pastor y Wise (2015), Saona (2016), Vianna y Mollick (2018), Williams (2018), Restrepo (2019), Yakovlev (2019), Onder y Ozyildrim (2019).

En este sentido, Aali-Bujari et al. (2017) estudian el impacto de la capitalización de mercado de valores y el spread bancario en el crecimiento económico, en las principales economías de América Latina durante el período 1994-2012. Para ello, estiman un modelo de datos de panel con el Método Generalizado de Momentos tanto en sistema como en diferencias. Sus resultados indican que el crecimiento económico en los países estudiados es afectado positivamente por la capitalización bursátil y negativamente por el spread bancario. La mayoría de los trabajos en este campo, apuntan a una relación positiva entre desarrollo financiero y la actividad económica. Los autores coinciden en que la relación existente entre el desarrollo financiero y el crecimiento económico debe ser considerada al momento de evaluar elementos que promuevan el crecimiento económico sostenido y a largo plazo de las naciones. Por otro lado, Pradhan et al. (2017) estudian la relación causal entre desarrollo financiero, penetración de las TIC y crecimiento económico en once países, en el periodo 1961-2012, utilizan datos panel, encuentran que existe causalidad de Granger tanto a corto, como a mediano plazo entre a variables: penetración de TIC, desarrollo del sector financiero y crecimiento económico para los países considerados en la muestra.

En ésta investigación se evalúa el impacto del desarrollo financiero en el crecimiento económico en las economías de América Latina. Específicamente se examina el impacto de las principales variables, como: el número de sucursales bancarias por cada 100000 adultos y el número de cajeros automáticos por cada 100000 adultos; en el crecimiento del PIB real per cápita, en el período 2004-2018. Para ello se utiliza el análisis de datos de panel con información del Banco 
Mundial a fin de encontrar evidencia sobre los nexos del desarrollo financiero y el crecimiento económico. Asimismo, con base en el modelado econométrico propuesto, se establecerán varias recomendaciones que permitan incrementar los niveles de bienestar en la región.

Con referencia al estado actual del tema, el presente trabajo se distingue en que: 1) se concentra en la región de América Latina, haciendo énfasis en las siete principales economías (Argentina, Brasil, Chile, Costa Rica, Ecuador, México, Perú y Uruguay); 2) se tiene una mayor disponibilidad de datos con respecto al pasado, 3) se realiza un análisis de datos de panel, que permite utilizar una mayor cantidad de países, de variables y de períodos; también permiten corregir problemas econométricos. Asociado a lo anterior, se desprende la hipótesis de este trabajo de investigación, que el desarrollo financiero tiene un impacto positivo en el crecimiento económico en América Latina, y por tanto las variables asociadas al desarrollo financiero (el número de sucursales bancarias por cada 100000 adultos y el número de cajeros automáticos por cada 100000 adultos), atendidas podrían jugar un rol importante en el crecimiento económico en la región.

El objetivo de ésta investigación es evaluar el impacto del desarrollo financiero en el crecimiento económico en América Latina, específicamente, se analiza el rol el número de sucursales bancarias por cada 100000 adultos y el número de cajeros automáticos por cada 100000 adultos, en el crecimiento económico en ocho países² , en el periodo 2004-2018; para ello, se utiliza análisis de datos panel para naciones latinoamericanas, con información del Banco Mundial disponible en el 2020 y los paquetes Stata e Eviews. El documento se organiza de la siguiente manera, el segundo apartado se dedica a la revisión de literatura del desarrollo financiero y crecimiento económico, el tercero corresponde a descripción estadística de variables, el cuarto a la descripción teórica del modelo, en la quinta sección se analizan los resultados econométricos. Por último, se presentan las conclusiones y referencias de la investigación.

\section{Desarrollo financiero y crecimiento económico.}

La relación entre desarrollo financiero y crecimiento económico es relevante, Wicksell (1934) encuentra que el sistema bancario determina la tasa de interés en el mercado y que la demanda de crédito es el motor del crecimiento económico. Los intermediarios financieros canalizan los ahorros a los proyectos de inversión más productivos, identifican a los mejores empresarios, las mejores tecnologías, reducen los riegos asociados a la innovación, contribuyen a la eficiencia económica, promueven el progreso tecnológico y el crecimiento económico.

Por otro lado, De Gregorio (1996) discute sobre las restricciones crediticias, por un lado incrementan el ahorro agregado, y por lo tanto pueden contribuir al crecimiento económico. En este sentido, argumentan que las restricciones de crédito también tienen efectos negativos sobre la acumulación de capital humano y por tanto sobre el crecimiento. Asimismo, el Banco Mundial Banco Mundial afirma que alrededor de 2,500 millones de personas no utilizan servicios financieros formales y el 75\% de los pobres no tiene cuenta bancaria, por lo cual esta institución considera que el desarrollo financiero es un factor clave para reducir la pobreza e impulsar la prosperidad. Por otro lado, el sistema financiero ayuda a movilizar el ahorro, asignar el capital, contribuye a un mercado de valores más robusto, eficiente, que a su vez promueve la actividad económica. En otro sentido,

\footnotetext{
${ }^{2}$ Las ocho naciones objetos de este estudio, son: Argentina, Brasil, Chile, Costa Rica, Ecuador, México, Perú y Uruguay.
} 
analizan una muestra de datos de varios países y encuentran una relación positiva entre desarrollo financiero y crecimiento económico a largo plazo, sin embargo en países donde se ha presentado crisis financieras, encuentran una relación negativa de corto plazo.

La estructura del sistema bancario de cada país tiene implicaciones en los resultados del desarrollo financiero, en la medida que existe una mayor cantidad de participantes en el sector bancario, los usuarios de servicios financieros tendrán mayor acceso a cuentas de depósito, préstamos, sucursales, cajeros automáticos y productos financieros en general. Rodríguez y López (2009), estudian la relación existente entre el desarrollo financiero y el crecimiento económico en México, mediante series de tiempo, encuentran que el desarrollo financiero impacta de forma positiva en el crecimiento económico y que existe una relación bidireccional entre ambas variables. El funcionamiento del sistema financiero y el crecimiento económico se analiza en Levine (2004), estudia la causalidad entre el sector financiero y la actividad económica, encuentra que los sistemas financieros más desarrollados ayudan a reducir las restricciones financieras externas que enfrentan las empresas, esto es un mecanismo a través del cual el desarrollo financiero influye en el crecimiento económico. El desarrollo del sector financiero contribuye a relajar las restricciones de financiamiento externo de las empresas y con ello promueve el crecimiento económico, Rajan y Zingales (1998) estudian una muestra de 36 industrias pertenecientes a 48 países y concluyen que el desarrollo del sector financiero impulsa la actividad económica.

En este sendero, Aali-Bujari et al. (2016) evalúan el impacto de los mercados de derivados sobre el crecimiento económico en las seis principales economías del mundo (Unión Europea, Estados Unidos, Japón, China, India y Brasil) durante el período 2002-2014, estiman un modelo de datos de panel con el Método Generalizado de Momentos), encuentran de manera empírica que los mercados de derivados de las economías estudiadas influyen positivamente en su crecimiento económico. Asimismo proporcionan varias recomendaciones útiles para promover el uso de los mercados de derivados con el fin de impulsar el crecimiento económico. Asimismo, el sector financiero promueve el crecimiento económico por medio del aumento de la tasa de acumulación de capital, King y Levine (1993) después de estudiar una muestra de alrededor de ochenta países y hallan que el sector financiero ayuda a mejorar la eficiencia económica y está fuertemente correlacionado con el crecimiento real del ingreso per cápita. Asimismo, la evidencia empírica presentada por Levin y Zervos (1998), sugiere que el desarrollo del mercado valores está fuertemente asociado y correlacionado positivamente con el crecimiento económico a largo plazo.

El desarrollo del sector financiero incentiva la innovación acelerando el progreso tecnológico y por tanto el crecimiento económico, De la Fuente y Marín (1996), analizan la interacción de los intermediarios financieros, la acumulación de capital físico, el progreso tecnológico y el crecimiento económico. Argumentan que el crecimiento económico se sustenta en el desarrollo de nuevas variedades de bienes intermedios ${ }^{3}$, la innovación es un riesgo y la probabilidad de éxito depende de las acciones de los empresarios, los intermediarios financieros negocian contratos de incentivos a los innovadores, y permiten una mejor distribución del riesgo, un mayor nivel de actividad innovadora, lo cual acelera el progreso tecnológico y el crecimiento económico.

\footnotetext{
${ }^{3}$ Se basan en los modelos schumpeterianos como Helpman y Grosman (1991), Aghion y Howitt (1992).
} 
Asimismo, Pérez-Akaki y Fonseca-Soto (2017) utilizan técnicas de análisis econométrico espacial y encuentran una relación causal relevante y positiva entre la inclusión financiera y la pobreza en los municipios mexicanos. López-Mateo et al. (2018) estudian el papel del desarrollo del sistema financiero, analizan sus determinantes en México para el periodo 2001-2014. Utilizan modelos de datos panel, encuentran que el desarrollo del sistema financiero depende directamente de la concentración y rentabilidad bancaria. Por otro lado, Pessoa et al. (2019) estudian la relación entre desarrollo financiero y crecimiento económico en Brasil, utilizan datos de los estados brasileños, en el periodo 1995-2014, con datos panel, concluyen que existe una relación positiva y no lineal entre el desarrollo financiero y crecimiento económico.

\section{Estadística descriptiva de variables}

Los datos que se utilizan en este trabajo son obtenidos del (Banco Mundial, 2020)4, el Producto Interno Bruto real per cápita (PIB per cápita), es la variable dependiente y las variables independientes son: el número de sucursales bancarias y el número de cajero automáticos, el PIB real per cápita está expresado en dólares estadounidenses de la paridad del poder adquisitivo del 2010, el número de sucursales bancarias esta expresado en términos absolutos para cada 100000 adultos, el número de cajeros automáticos está expresado por cada 100000 adultos. Se dispone de datos de panel balanceado con el objetivo de estimar un modelo de datos panel, el periodo y número de países, se encuentra restringido por la disponibilidad de datos, el panel incluye ocho países de América Latina ${ }^{5}$ para el periodo entre 2004-2018. La muestra está restringida a las ocho economías cuya información está disponible. El cuadro 1, muestra las notaciones de las variables utilizadas en este trabajo, que son el producto interno bruto real per cápita, número de sucursales, número de cajeros automáticos, así como sus promedios, las desviaciones estándar, los niveles máximos y mínimos.

Cuadro 1. Estadística de variables

\begin{tabular}{|l|c|c|c|c|l|}
\hline Variable & Notación & Promedio & Desviación & Mínimo & Máximo \\
\hline Producto Interno Bruto real per cápita & PIBper & 9295.739 & 3047.283 & 3603 & 15130.15 \\
\hline Número de sucursales & ns & 14.28232 & 4.38454 & 4.20973 & 22.96379 \\
\hline Número de cajeros & nc & 52.18296 & 32.07875 & 1.86135 & 122.7815 \\
\hline
\end{tabular}

Fuente: elaboración propia con datos de Banco Mundial.

El cuadro 1, muestra la estadística de las variables utilizadas en este trabajo. El PIB real per cápita promedio para los 8 países que se estudian es 9295.73 USD al año, desviación estándar de 3047.28 USD, el menor ingreso real per cápita corresponde a Perú (2004), el mayor ingreso per cápita corresponde a Chile (2018). Respecto al número de sucursales bancarias, el promedio es de 14.28 sucursales por cada 100000 adultos, la desviación estándar es de 4.38 sucursales por cada 100000 adultos, el país con menos sucursales por cada 100000 adultos corresponde a Perú (2004), mientras que Costa Rica (2011) tuvo el mayor número de sucursales por cada 100000 adultos, que es de 22.96.

\footnotetext{
${ }^{4}$ Indicadores de Banco Mundial disponibles en 2020.

${ }^{5}$ Los países son: Argentina, Brasil, Chile, Costa Rica, Ecuador, México, Perú y Uruguay. El número de países está limitado por la disponibilidad de datos país en la base de datos del Banco Mundial.
} 
Por otro lado, el número de cajeros promedio es 52.18 cajeros por cada 100000 adultos, la desviación estándar es de 32.07 cajeros por cada 100000 adultos, el país con menos cajeros corresponde Argentina (2004), con 1.86 cajeros por cada 100000 adultos, mientras que el país con más cajeros automáticos corresponde a Perú 2015. A continuación se muestran los resultados del análisis gráfico, que relaciona la variable dependiente producto interno bruto real per cápita, con las variables del sector financiero como objetos de este estudio como, número de sucursales por cada 100000 adultos y número de cajeros por cada 100000 adultos en las economías de América Latina.

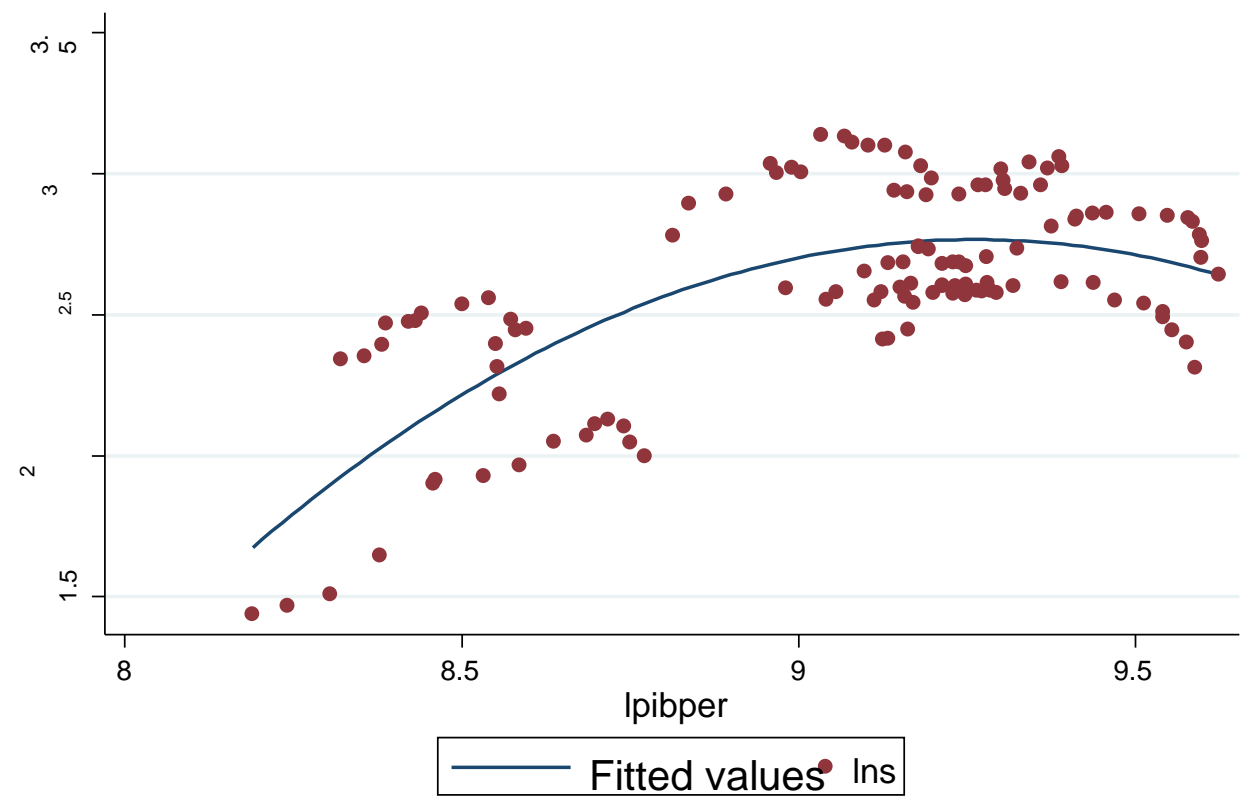

Grafica 1. Dinámica del número de sucursales y PIB real per cápita Elaboración propia con datos de Banco Mundial.

La gráfica 1, muestra la dinámica del número de sucursales bancarias relacionada con el PIB real per cápita para las ocho economías analizadas de América Latina (Argentina, Brasil, Chile, Costa Rica, Ecuador, México, Perú y Uruguay) durante el periodo 2004-2018, se observa una relación positiva, aumentos en el número de sucursales bancarias por cada 100000 adultos están asociados con el incremento del PIB real per cápita. Por otro lado, esto sugiere que los países con un mayor número de sucursales bancarias por cada 100000 adultos están en mejores condiciones para elevar su PIB real per cápita, asimismo las economías con mayor PIB real per cápita tienen la posibilidad de contar con mayor número de sucursales bancarias por cada 100000 adultos en las economías objeto de estudio, esto es un argumento a favor de que el desarrollo financiero está asociado de forma positiva con el crecimiento económico. 


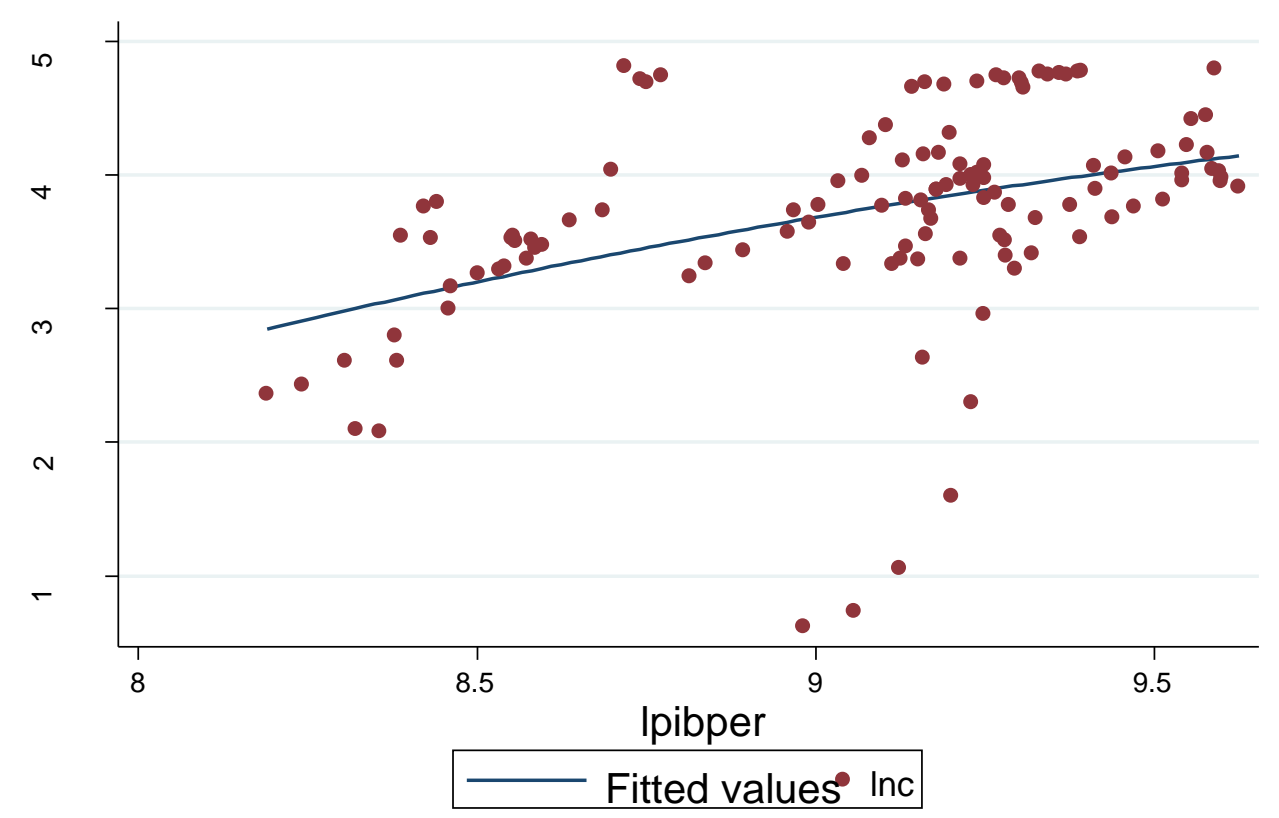

Gráfica 2. Comportamiento del número de cajeros y PIB real per cápita. Elaboración propia con datos de Banco Mundial.

En la gráfica 2 se representan gráficamente los datos del número de cajeros automáticos y el producto interno bruto real per cápita en ocho economías de América Latina en el periodo 20042018. La gráfica muestra la dispersión de las observaciones y la línea de tendencia ascendente que indica una relación positiva entre las variables, se puede argumentar que los países con un mayor número de cajeros automáticos por cada 100000 adultos están en mejores condiciones para elevar su PIB real per cápita. Además, es más probable que los países con un mayor PIB real per cápita ostentan un mayor número de cajeros automáticos por cada 100000 adultos. En resumen, la gráfica 2, apunta a la idea de que el desarrollo financiero impulsa el producto interno bruto real per cápita y a su vez el producto interno bruto real per cápita afecta positivamente al desarrollo financiero.

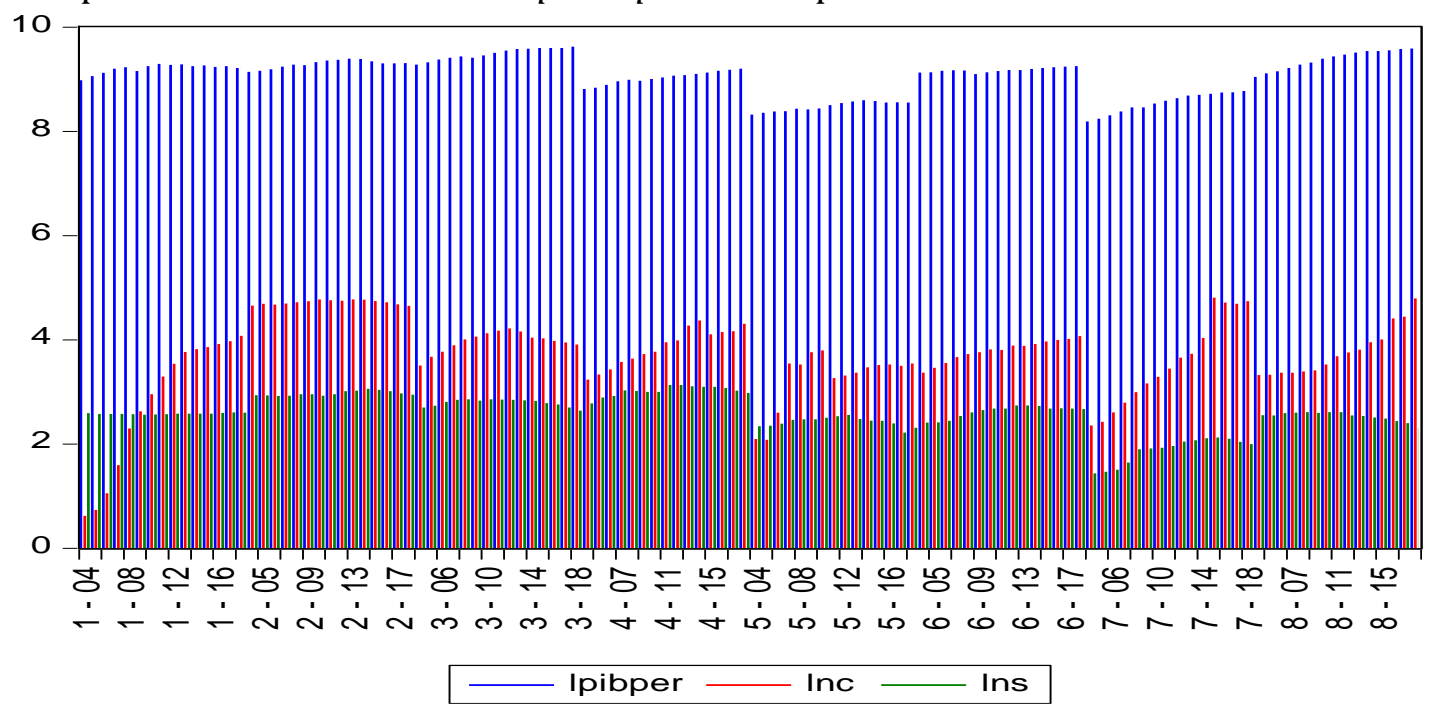

Grafica 3. Dinámica del número de sucursales, número cajeros y PIB real per cápita.

Elaboración propia con datos de Banco Mundial. 
La Grafica 3, muestra la dinámica del número de sucursales bancarias por cada 100000 adultos, el número de cajeros automáticos por cada 100000 adultos y PIB real per cápita en las ocho economías objeto de estudio, se observa que aumentos en el número de sucursales bancarias y en el número de cajeros automáticos están asociados con el aumento en PIB real per cápita. Mientras que disminuciones en número de sucursales bancarias y en el número de cajeros automáticos están asociados con la disminución en el PIB real per cápita. En resumen las tres gráficas indican que las variables utilizadas como proxys del desarrollo financiero tiene un impacto positivo en el aumento del ingreso real per cápita en las economías latinoamericanas que son objetos de ésta investigación. La mayor parte de los trabajos que estudian la relación del desarrollo financiero y el crecimiento económico predicen que existe una correlación positiva como lo señalan las gráficas 1,2 y 3 y refuerzan este tipo de pensamiento.

\section{Especificación econométrica del modelo de datos panel}

La investigación aplicada crece y cada vez más, utiliza con mayor frecuencia el análisis de datos panel, ya que es muy útil para la investigación aplicada, los datos de panel son una muestra de características que tienen los individuos, familias, empresas, industrias, países, a lo largo del tiempo. El panel relaciona datos de series de tiempo y de cortes transversal ${ }^{6}$. El modelo general que se pretende estimar es:

$$
y_{i t}=\alpha y_{i t-1}+\beta X_{i t}+u_{i t}
$$

Las estimaciones de datos panel se realizan por distintos métodos, por mínimos cuadrados ordinarios (MCO) y por otro lado, mediante el anidamiento de los datos: modelo de efectos fijos (EF) y modelo de efectos aleatorios (EA), como modelos alternativos a la regresión agrupada. El uso de datos de panel presenta varias ventajas porque tiene mayor número de observaciones, más y mejor información, admite mayor número de variables y menos multicolinealidad entre datos de las variables explicativas, mayor eficiencia en la estimación, y se puede hacer un seguimiento de cada país, individuo o unidad de observación. También alivia el problema de variables omitidas, ya que se pueden eliminar por diferencia las que se mantienen constantes en el tiempo ${ }^{7}$. Por otro lado, el uso de datos panel presenta sus desventajas, ya que los datos son más complejos, se enfrentan a otro tipo de problemas, que son diferentes a los problemas comunes en datos de series de tiempo y de datos de corte trasversal. El modelo de efectos fijos implica menos suposiciones sobre el comportamiento de los residuos. Supone que el modelo a estimar es ahora:

$$
y_{i t}=\alpha y_{i t-1}+\beta X_{i t}+\varepsilon_{i t}
$$

\footnotetext{
${ }^{6}$ Información de varios países, empresas, individuos, a lo largo del tiempo.

7 Para profundizar en el tema sobre las bondades y limitaciones del uso datos panel, el lector puede revisar Baltagi (1995).
} 
Consideramos que $\varepsilon_{i t}=v_{i}+u_{i t}$, luego reemplazando en (3.2) queda:

$$
y_{i t}=\alpha y_{i t-1}+\beta X_{i t}+v_{i}+u_{i t}
$$

Es decir, supone que el error $\varepsilon_{\text {it }}$ se divide en dos partes, una parte fija, constante para cada individuo o país $v_{i}$ y otra aleatoria $u_{i t}$ que cumple los requisitos MCO $\left(\varepsilon_{i t}=v_{i}+u_{i t}\right)$, lo que es equivalente llevar a cabo una regresión general y asignar, a cada individuo o país un punto de corte diferente (ordenada distinta). Asimismo, el modelo de efectos aleatorios tiene la misma especificación que el de efectos fijos, sin embargo $v_{i}$ en lugar de ser una constante para cada país $y$ tener valor fijo a lo largo del tiempo es una variable aleatoria con un valor medio $\mathrm{v}_{\mathrm{i}} \mathrm{y}$ una varianza $\operatorname{Var}\left(\mathrm{v}_{\mathrm{i}}\right) \neq 0$. Es decir la especificación del modelo es igual a (3.3).

$$
y_{i t}=\alpha y_{i t-1}+\beta X_{i t}+v_{i}+u_{i t}
$$

La diferencia ahora, es que $v_{i}$ es una variable aleatoria.

\section{Análisis de resultados}

El objetivo de esta sección es construir un modelo de datos panel, en base a la información disponible en el 2020, proporcionada por el banco mundial, y que el modelo permita analizar la relevancia que tiene el desarrollo financiero en el crecimiento económico en las economías latinoamericanas objetos de ésta investigación en el periodo 2004 - 2018. Nuestro estudio se centra en una muestra de ocho países latinoamericanos: Argentina, Brasil, Chile, Costa Rica, Ecuador, México, Perú y Uruguay; tomando en cuenta las variables, producto interno bruto real per cápita, número de sucursales bancarias por cada 100000 adultos y número de cajeros automáticos por cada 100000 adultos, contando con 120 observaciones, se estimó un panel balanceado en el paquete econométrico Stata, los principales resultados, se presentan en el siguiente cuadro.

Cuadro 2. Modelos de datos panel

\begin{tabular}{|l|c|c|c|}
\hline Variable Dependiente: Ipibper & BE & EF & EA \\
\hline lns & $\begin{array}{c}0.6179728 \\
(0.190)\end{array}$ & $\begin{array}{c}0.1921195 \\
(0.016)\end{array}$ & $\begin{array}{c}0.2096172 \\
(0.006)\end{array}$ \\
\hline lnc & $\begin{array}{c}0.0694066 \\
(0.803)\end{array}$ & $\begin{array}{c}0.1241995 \\
(0.000)\end{array}$ & $\begin{array}{c}0.123195 \\
(0.000)\end{array}$ \\
\hline $\boldsymbol{R}^{\mathbf{2}}$ & 0.3800 & 0.5010 & 0.5008 \\
\hline ML BP & & & Prob>Chi2 $=0.0575$ \\
\hline Hausman & & & Prob>Chi2 $=0.6073$ \\
\hline Número de países & 8 & 8 & 8 \\
\hline Numero de observaciones & 120 & 120 & 120 \\
\hline
\end{tabular}

Entre paréntesis el error estándar correspondiente.

Fuente: elaboración propia con estimaciones en Stata

${ }^{8}$ Se analiza una muestra de ocho economías, cuya información está disponible en Banco Mundial en 2020. 
El cuadro 2, presenta los resultados de las estimaciones de modelos de datos panel: modelo de corte de trasversal (BE), modelo de efectos fijos (EF) y modelo de efectos aleatorios (EA). La primera columna señala que la variable dependiente es el logaritmo del producto interno bruto real per cápita, las variables explicativas el logaritmo del número de sucursales bancarias por cada 100000 adultos y el logaritmo de número de cajeros automáticos por cada 100000 adultos, el coeficiente de determinación $\left(\mathrm{R}^{2}\right)$, la prueba del multiplicador de Lagrange (ML BP), posteriormente la prueba de Hausman, número de países y número de observaciones de la muestra. La segunda columna del cuadro 3.2, muestra los resultados de las estimaciones de corte trasversal (BE) ${ }^{9}$, los coeficientes de las variables: logaritmo del número de sucursales bancarias y el logaritmo de número de cajeros automáticos presentan signos positivos esperados, mientras que ninguno de los coeficientes es significativo, el modelo presenta una $\mathrm{R}^{2}=0.38$, el número de países es 8 y se cuenta con 120 observaciones.

Por otro lado, la tercera columna presenta el modelo de efectos fijos que indica coeficientes positivos y significativos de ambas variables explicativas: logaritmo del número de sucursales bancarias y el logaritmo de número de cajeros automáticos, el modelo de efectos fijos presenta un coeficiente de determinación más alto en comparación con modelo de corte trasversal $\left(R^{2}=0.5010\right)$, el número de países es 8 y se cuenta con 120 observaciones. Asimismo, la cuarta columna presenta el modelo de efectos aleatorios, las estimaciones señalan coeficientes positivos y significativos, tanto para el logaritmo del número de sucursales bancarias, como para el logaritmo de número de cajeros automáticos, el coeficiente de determinación del modelo de efectos aleatorios $\left(R^{2}=0.5008\right)$ es ligeramente menor respecto al modelo de efectos fijos.

Posteriormente se presenta la prueba del multiplicador de Lagrange que arroja un prob> chi $2=0.0575$, que indica que la estimación por efectos aleatorios es preferible a mínimos cuadrados ordinarios; asimismo se presenta la prueba de Hausman con prob $>$ chi2 $=0.6073$ indicándonos que el modelo de efectos aleatorios es preferible al modelo de efectos fijos. Por último, se indica el número de países es 8 y se cuenta con 120 observaciones. En síntesis, se llevaron a cabo estimaciones con diferentes métodos, para analizar la interrelación entre las variables financiera con el PIB real per cápita, se utilizan datos panel, modelos efectos fijos y efectos aleatorios y las pruebas de multiplicador de Lagrange y de Hausman, en este trabajo hallamos el modelo de efectos aleatorios es la preferido entre los modelos que se estiman en ésta investigación para explicar el impacto de las variables financieras al crecimiento económico en los países y durante el periodo que se analiza.

Las estimaciones indican que modelo de efectos aleatorios es el modelo de mejor ajuste entre los modelos de datos panel estimados, asimismo muestra que el aumento en el número de sucursales bancarias por cada 100000 adultos causa un incremento en el PIB real per cápita, por otro lado, un aumento del número de cajeros automáticos por cada 100000 adultos causa un incremento en el PIB real per cápita, en las economías que son objetos de estudio. Aunado a lo anterior, encontramos que el PIB real per cápita presenta mayor sensibilidad al número de sucursales bancarias por cada 100000 adultos, comparado con el número de cajeros automáticos por cada 100000 adultos.

\footnotetext{
${ }^{9}$ La estimación de corte trasversal utiliza las medias de las variables.
} 


\section{Conclusiones}

El objetivo de ésta investigación es analizar el impacto del desarrollo financiero en el crecimiento económico en América Latina y demostrar con evidencia empírica, la hipótesis de este trabajo, que el desarrollo financiero tiene un impacto positivo en el crecimiento económico, en concordancia con la mayor parte de la teoría en este campo. Efectivamente los resultados obtenidos señalan que existe un impacto positivo de número de sucursales bancarias por cada 100000 adultos en el incremento del PIB real per cápita, así como un impacto positivo del número de cajeros automáticos por cada 100000 adultos en incremento PIB real per cápita, en periodo analizado 2004-2018.

Mediante el análisis descriptivo, ésta investigación muestra que tanto número de sucursales bancarias por cada 100000 adultos, como el número de cajeros automáticos por cada 100000 adultos, tiene relación positiva con el PIB real per cápita, que el desarrollo financiero está ligado al crecimiento económico en la región de América Latina (Argentina, Brasil, Chile, Costa Rica, Ecuador, México, Perú y Uruguay), en el periodo que se analiza. Las estimaciones de modelos de datos panel presentan la importancia del desarrollo financiero (número de sucursales bancarias por cada 100000 adultos y número de cajeros automáticos por cada 100000 adultos) para el crecimiento económico en América Latina.

Todos los modelos estimados en el presente trabajo de investigación, tanto el modelo de corte trasversal, modelo de efectos fijos, como modelo de efectos aleatorios indican un impacto positivo del desarrollo financiero en el crecimiento económico de las economías que se analizan, por otro lado indican que el número de sucursales bancarias por cada 100000 adultos tiene mayor impacto en el crecimiento económico, en comparación con el número de cajeros automáticos por cada 100000 adultos. El desarrollo financiero a través de en el número de sucursales bancarias y de cajeros automáticos por cada 100,000 habitantes sí tiene efecto positivo en el crecimiento económico en América Latina aun cuando el Banco Mundial afirma que alrededor de 2,500 millones de personas no utilizan servicios financieros formales y el $75 \%$ de los pobres no tiene cuenta bancaria.

La presente investigación propone a los tomadores de decisiones en los países latinoamericanos buscar los instrumentos e incentivos adecuados para fomentar el desarrollo financiero (incrementando el número de sucursales bancarias por cada 100000 adultos y aumentando el número de cajeros automáticos por cada 100000 adultos), que inciden en el incremento de PIB real per cápita y por tanto en el crecimiento económico, empleo y bienestar de las familias. Estas medidas pueden estar orientadas al sector financiero, banca, cajas de ahorro, fondos de pensión, con incentivos fiscales y otros, de manera que promuevan el desarrollo financiero. Por otro lado, impulsar el sector financiero como medio para fomentar la actividad económica, empleo y con ello lograr mayores niveles de bienestar en la región. La investigación se limita a analizar el rol dos variables del desarrollo financiero (el número de sucursales bancarias y el número de cajeros automáticos) en el crecimiento económico, que son relevantes desde el punto de vista de resultados de ésta investigación, sin embargo recomienda para futuras investigaciones, explorar desde otras perspectivas el papel que juegan otras variables de desarrollo financiero en el crecimiento económico, así como el impacto del desarrollo económico en el desarrollo financiero. 


\section{Referencias}

[1] Aali Bujari, A., Venegas Martínez, F. y Pérez Lechuga, G. (2016). Impact of Derivatives Markets on Economic Growth in a some of the major World Economies. A Difference-GMM Panel Data Estimation (2002-2014). AESTIMATIO, The IEB International Journal of Finance, 12, 110-127. DOI:10.5605/IEB.12.6

[2] Aali Bujari, A., Venegas-Martínez, F., Pérez Lechuga, G. (2017). Impact of the Stock Market Capitalization and the Banking Spread in Growth and Development in Latin American: A Panel Data Estimation with System GMM. Contaduría y Administración, 62 (5), 1427-1441. https://doi.org/10.1016/j.cya.2017.09.005

[3] Aghion, P y Howitt, P. (1992). A Model of Growth through Creative Destruction. Econometrica, 60 (2), 323-351. DOI:10.3386/w3223

[4] Baltagi, B. (1995). Editors Introduction Panel Data. Journal of Econometrics, 68 (1), 1-4. https://doi.org/10.1016/0304-4076(95)90009-8

[5] Beck, T. y Levine, R. (2002). Industry Growth and Capital Allocation: Does Having a Market-Or BankBased System Matter? Journal of Financial Economics, 64 (2), 147-180. doi=10.1.1.708.8638\&rep=rep1\&type=pdf

[6] Bencivenga, V. Smith, R. y Starr, R. (1996). Equity Markets, Transactions Costs, and Capital Accumulation: An Illustration. World Bank Economic Review, 10 (2), 241-266. https://doi.org/10.1596/1813-9450-1456

[7] Bittencourt, M. (2012). Inflation and Economic Growth in Latin America : Some Panel Time-series Evidence. Economic Modelling, 29 (2), 333-340. DOI: 10.1016 / j.econmod.2011.10.018

[8] Blanco, L. (2013). Finance, Growth and Institutions in Latin America: what are the links. Journal of Economics, 50 (2), 179-208. DOI: 10.7764 / LAJE.50.2.179

[9] De Gregorio, J. (1996). Borrowing Constraints, Human Capital Accumulation and Growth. Journal of Monetary Economics, 37 (1), 49-71. https://doi.org/10.1016/0304-3932(95)01234-6

[10] De la Fuente, J. y Marín, J. (1996). Innovation, Bank Monitoring and Endogenous Financial Development. Journal of Monetary Economics, 38 (2), 269-302. https://doi.org/10.1016/S03043932(96)01277-9

[11] Golsmith, R. (1969). Financial Estructure and Development. New Haven: Yale University Press.

[12] Grosman, G y Helpman, E. (1991). Quality Ladders in the Theory of Growth. Review of Economic Studies, 58(1), 43-61. https://doi.org/10.2307/2298044.

[13] Guidotti, P. (2007). Global Finance, Macroeconomic Performance, and Policy Response in Latin America: Lessons from the 1990s. Journal of Applied Economics, 10(2), 279-308. DOI: 10.1080 / 15140326.2007.12040491

[14] .King, R. y Levine, R. (1993). Finance and Growth: Schumpeter might be Right. The World Bank, Policy Research Working Paper Series 1083. DOI: 10.2307 / 2118406

[15] Levine, R. (2004). Finance and Growth: Theory and Evidence. NBER Working, papers 10766, National Bureau of Economic Research, Inc. https://www.nber.org/papers/w10766.pdf

[16] Levine, R. y Zervos, S. (1998). Stock Markets. Banks and Economic Growth. American Economic Review, 88 (3), 537-558. https://econpapers.repec.org/paper/wbkwbrwps/1690.htm

[17] López-Mateo, C., Ríos-Manríquez, M. y Cárdenas-Alba, L. (2018). Competencia, Inclusión y Desarrollo del Sistema Financiero en México. Investigación Administrativa, 47 (121), 1-22. http://www.scielo.org.mx/pdf/ia/v47n121/2448-7678-ia-121-00001.pdf

[18] McKinon, R. (1973). Money and Capital in Economic Development. Washington, D. C. Brookings Institution. https://doi.org/10.2307/1239377. 
[19] Nazmi, N. (2005). Deregulation, Financial Deepining and Economic Growth: The Case of Latin America. Quarterly Review of Economics and Finance, 45(2), 447-459. https://ideas.repec.org/a/eee/quaeco/v45y2005i2-3p447-459.html

[20] Onder, Z., y Ozyildirim, S. (2019). Foreing Banks and Short-term Macroeconomic Fluctuations: Do Financial Development and Regions Matter? Economic Systems, 43 (1), 63-76. https://dx.doi.org/10.1016/j.ecosys.2018.08.007

[21] Pastor, M., y Wise, C. (2015). Good Bye Financial Crash, Hello Financial Eclecticism: Latin American Responses to the 2008-09 Global Financial Crisis. Journal of International money and Finance, 52, 200217. https://ideas.repec.org/a/eee/jimfin/v52y2015icp200-217.html

[22] Pérez Akaki, P. y Fonseca Soto, M. (2017). Análisis Espacial de la Inclusión Financiera y su relación con el Nivel de Pobreza en los Municipios Mexicanos. Revista Mexicana de Economía y Finanzas, 12 (1), 43 62. http://www.scielo.org.mx/pdf/rmef/v12n1/2448-6795-rmef-12-01-00043.pdf

[23] Pessoa, F.C., Braga, M. y Morais, G. (2019), Crecimiento Económico y Desarrollo Financiero en Brasil: Investigación apatir del Modelo de Regresión Flexible. Revista de la CEPAL, 128, 101-123. https://repositorio.cepal.org/bitstream/handle/11362/44739/1/RVE128_Braga.pdf

[24] Pradhan, R.P., Arvin, M., Nair, M., Brenett, S. y Bahmani, S. (2017). ICT-Finance-Growth nexus: Empirical Evidence from the Next-11 countries. Cuadernos de Economía, 40 (113), 115-134. https://doi.org/10.1016/j.cesjef.2016.02.003

[25] Rajan R. y Zingales, L. (1998). Financial Dependence and Growth. American Economic Review, 88 (3), 559-586. https://econpapers.repec.org/paper/nbrnberwo/5758.htm

[26] Restrepo, F. (2019). The Effects of Taxing Bank Transactions on Bank Credit and Industrial Growth: Evidence from Latin America. Journal of International Money and Finance , 93, 335-355. DOI: 10.1016 / j.jimonfin.2019.02.005

[27] Rodríguez, D. y López, F. (2009). Desarrollo Financiero y Crecimiento Económico en México. Problemas del Desarrollo: Revista Latinoamericana de Economía, 40 (159), 39-60. http://www.scielo.org.mx/pdf/prode/v40n159/v40n159a3.pdf

[28] Saona, P. (2016). Intra-and Extra-Bank Determinants of Latin America Banks' Profitability. International Review of Economics \& Finance, 45, 197-214. D0I: 10.1016 / j.iref.2016.06.004

[29] Schumpeter, J. (1954). History of Economic Analysis. Oxford: Oxford University Press.

[30] Tabak , B., Fazio, D. M., y Cajuero, D. O. (2013). Systemically important Banks and Financial Stability: The case of Latin America. Journal of Banking \& Finance, 37 (10), 3855-3866. DOI: 10.1016 / j.jbankfin.2013.06.003

[31] Venegas-Martínez, F. (1999). Crecimiento Endógeno, Dinero, Impuestos y Deuda Externa. Investigación Económica, 59 (229), 15-36. http://www.scielo.org.mx/pdf/ineco/v59n229/0185-1667-ineco-59229-15.pdf

[32] Vianna, A. C., y Mollick, A. V. (2018). Institutions: Key variable for Economic Development in Latin $\begin{array}{lllll}\text { America. Journal of Economics and } & \text { Business, }\end{array}$ https://doi.org/10.1016/j.jeconbus.2017.12.002

[33] Wicksell, K. (1934). Lectures on Political Economy. London: Routledge.

[34] Williams, K. (2018). Has the Finance -Growth link been broken? Panel Data Evidence from Latin America and the Caribbean. Economia, 19 (3), 404-423. https://doi.org/10.1016/j.econ.2018.10.001

[35] Yakovlev, P. (2019). Latin América: Is a spurt in Development Possible? World Economy and International Relations, 63 (3), 94-103. DOI: 10.20542/0131-2227-2019-63-3-94-103 